Zusammenhang zitiert werden: „The ambition and opportunism inherent, to various degrees, in the political vocation have been behind most of the adjustments, alterations, and adaptations that have marked the careers of men and parties in Ceylon since independence" (S. 289). Diese Frage führt bereits zu dem Kernproblem, ob das europäische Parteiensystem in den Entwicklungsländern überhaupt sinnvoll ist. Die geistesgeschichtliche Situation des Zusammenbruchs des abendländischen Weltbildes christlich-humanistischer Prägung ist der Hintergrund, auf dem sich der europäische Staat der Neuzeit mit seinen Parteien eingerichtet hat. Ist ein entsprechender Hintergrund für Ceylon gegeben? Nur wenn man diese Frage bejahen kann, d. h. wenn man unter den Firnis der englischen Kolonialherrschaft blickt und dort ähnliche Strukturen wie im Europa des 19. Jahrhunderts entdecken würde, könnte man eine Übertragung des westlichen Systems auf die Dauer für möglich halten. Gerade für diese Frage hätte der Verfasser in dem drei Jahre vor Erscheinen seines Buches 1966 in Deutschland veröffentlichten 1. Band des Standardwerkes von Heinz Bechert über Staat und Buddhismus eine Fundgrube par excellence zur Hand gehabt. Ein großer Teil des historischen Materials, das Woodward vorbringt, ist auch bei Bechert zu finden, jedoch mit zwei Vorzügen: Bechert berücksichtigt auf Grund seiner Sprachkenntnisse auch ausgiebig singhalesische Quellen, während Woodward nicht eine einzige nichtenglische Quelle angibt; ferner setzt sich Bechert mit der Eigenart der ceylonesischen Auffassungen über Staat und Gesellschaft auseinander, während Woodward alles rein vom westlichen Standpunkt aus betrachtet und die eigentliche Alternative des Ein- oder Zwei- oder Mehrparteiensystems, nämlich ein KeinParteien-System, nur unter dem $\mathrm{Ge}-$ sichtspunkt der unabhängigen Kandidaten und der Notabeln-Struktur erwähnt, d. h. wiederum unter rein westlichen Kategorien.

Hellmuth Hecker
HENRY Bienen

\section{Tanzania -}

Party Transformation and Economic

Development

Expanded Edition, Princeton UP 1970.

XXVIII, $506 \mathrm{~S}$.

G. ANDREW MAguire

Toward „Uhuru“ in Tanzania

The Politics of Participation,

Cambridge 1969, XXIX + 403 S.

Tansania ist unter dem Blickwinkel der drei friedlich miteinander um die Weltherrschaft konkurrierenden Weltmächte spezifisch interessant wegen einer einzigartigen chinesischen Präsenz (Eisenbahn nach Sambia!). Hiervon ist allerdings in beiden Büchern nicht die Rede, wenn man von der Erwähnung chinesischer Ausbilder (unter anderen!) in der Armee durch Bienen (378) absieht.

$\mathrm{B}$ i e $\mathrm{n}$ e $\mathrm{n}$ beschäftigt sich als erstes ausführlich mit Vorgeschichte und Entstehung der "Tanganyika African National Union" (= TANU) und ihres Führers, des „Vaters der Nation" (158; 164) Nyerere. Diese Partei ist seit 1963 (176) nicht mehr nur de facto, sondern auch de jure einzige Partei $(232 ; 239 ; 245$; 381). Die Begründung dieser Einzigkeit hat sich Nyerere selbst angelegen sein lassen: Neu hieran ist die These, daß das Einparteien-System demokratischer sei als alle anderen (228 ff.), jedenfalls wenn die eine Partei die Partei des ganzen Volkes und deswegen eine offene sei (beides zum Unterschied von einer Elite-Partei), ohne daß sie allerdings deswegen mit der Nation identisch wäre (242). Da Einzigkeit an sich das ausschließliche Wesensmerkmal des Modernen Staates ist (keine Staaten im Staate!), deutet die Einzigkeit der TANU darauf hin, daß sie aufgaben- und arbeitsmäßig weithin der Staat ist. Das erste zeigt sich vor allem im Zusammenhang mit der Bildung von Nation und Allgemeinheit. Zwar kennt Tansania weder „strong tribes" noch "pronounced regionalism" (197) und auch keinen Gegensatz zwischen Christen und Muslimen (188). Aber diese Vorzüge ändern nichts daran, daß auch in Tansania erst einmal der letzte Bewohner staatlich $\mathrm{zu}$ erfassen 
und der letzte Winkel staatlich zu durchdringen ist (254; vor allem 334 ff.: TANU Tries to Reach the Villages). $\mathrm{Da}$ TANU $z \mathrm{u}$ beidem besser befähigt ist, fällt ihr diese an sich staatliche Aufgabe zu (99; 309). Ihr ist ferner die Erweckung eines quantitativ und qualitativ allgemeinen Staatsbürgertums anvertraut (204). Die Partei ist es ferner, die dem Staat zur Autorität verhilft (310), ihn legitimiert (309) und seinen Gesetzen den Gehorsam der Bürger sichert, weil der Staat der Staat der Partei ist, der das Volk anhängt (309; vgl. auch 343: Die Eine Partei als „enforcer of central edicts in the country side"). Trotzdem läßt sich eine unpopuläre Politik noch nicht durchsetzen (347), und selbst TANU ist zu schwach, um die Wirtschaft lenken zu können. Angesichts solcher Identität der Funktionen nimmt es nicht wunder, daß man zahlreichen organisatorischen und personalen Verschränkungen zwischen Staat und Partei begegnet (etwa 90, 120 usw.). Hier wohl am bemerkenswertesten ist es, daß die Staatsminister sich vor dem Parteikongreß rechtfertigen müssen wie vor einem Parlament (174). Durch alles dies verflüchtigt sich der Unterschied zwischen Staat und Partei für die Bevölkerung (128).

Die Partei ist vor allem das Subjekt der Modernisierung, der "agent of chan-

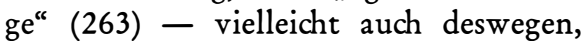
weil Nyerere mit Nachdruck die Notwendigkeit betont, die Modernisierung $\mathrm{zu}$ beherrschen, statt sich von ihr beherrschen zu lassen (418). Diese Modernisierung gilt vor allem der Wirtschaft, und zwar im Zeichen eines "Afrikanischen Sozialismus", über den zwar oft berichtet wird, ohne daß aber deutlich geworden wäre, was eigentlich damit gemeint ist (vgl. vor allem $406 \mathrm{ff.:}$ „The Arusha Formulations"). Offenbar soll eine „sozialistische" Nation trotz Industrialisierung vor dem Primat der Wirtschaft (262 f.; 414) und vor der Vorherrschaft des erwerbswirtschaftlichen Motivs (449) bewahrt werden. Verfahrensmäßig hat sich die Wirtschaftspolitik der Planung (vor allem $281 \mathrm{ff} .:$,The Formulation of the Five Year Plan") innerhalb einer Gemischten Wirtschaftsverfassung (248) verschrieben. Über alles dies erfährt man mancherlei interessante Einzelheiten, etwa die Auffassung, daß eine Planungsbehörde zu den Symbolen der Staatlichkeit gehört (280) und daß es nicht zuletzt auf die Erwekkung eines Ethos der Arbeit ankommt (161).

*

Maguire beschränkt seine Darstellung räumlich auf Sukuma, eine nördliche Landschaft, die an den Victoriasee angrenzt. Indem die gesamtheitlichen Vorgänge in dieser regionalen Brechung vorgeführt werden, gewinnen sie an Farbe und Greifbarkeit. Das gilt vor allem für die ersten zwei Drittel des Buches, die der unmittelbaren Vorgeschichte der Erlangung der Selbständigkeit gewidmet sind. Sukuma zeigt als erstes, wie das Prinzip der indirect rule zum Nachteil nicht nur des Kolonialvolkes, sondern vor allem auch der Kolonialherren ausschlagen kann (122). Als sich nämlich das Verlangen nach Unabhängigkeit immer stärker äußert (101; über die Anfänge eines Verlangens nach vollständiger Selbständigkeit vgl. $132 \mathrm{ff}$.; 151 ff.; 180) und diese Unzufriedenheit sich in der Urgestalt der TANU, der TAA (= Tanganyika African Association) organisiert, da verweigern die englischen Beamten diesen neuen politischen Organisationen das Gespräch mit der Begründung, etwas Derartiges sei „Syndikalismus" (101), und verweisen diese Bewegung auf den „Dienstweg“, nämlich an die Häuptlinge, die die Kolonialmacht aus Stammeshäuptern in Lokalinstanzen verwandelt hat. Hierdurch steigert sich nicht nur die Unzufriedenheit -, es leidet hierunter vor allem das Ansehen der Häuptlinge, die die Erfüllung von Forderungen verweigern, die sie eine Stellung kosten würden, die sie der Kolonialherrschaft verdanken. Alles dies wirkt um so unbegreiflicher als bei den Engländern die Überzeugung vorwaltet, daß selbst die tüchtigste Kolonialverwaltung die Dekolonisierung nicht würde aufhalten können (135). 
$\mathrm{Zu}$ denjenigen Organisationen, denen die Engländer das Gespräch verweigern, gehört vor allem die TAA, die später in TANU umbenannt wird, damit der politische Charakter der Bewegung im Namen zum Ausdruck gelangt (170). Diese Vorgänge werden im Lichte der Region Sukuma eingehend und vor allem unter genauer Vorstellung der beteiligten Personen berichtet. Am interessantesten scheint mir die Darstellung der Entstehung des ländlichen Genossenschaftswesens zu sein, und zwar zunächst in Gestalt von Absatzgenossenschaften der Baumwollpflanzer. Diese Bewegung wird dadurch ausgelöst und zum Erfolg geführt, daß ein Empfinden für die Ausbeutung durch die asiatischen Baumwoll-Aufkäufer erwacht (81 ff. vor allem 82;86;89; 109; 164), daß man die Möglichkeit der Selbsthilfe erkennt und sich, hierzu angehalten vor allem durch die bewunderungswürdige Energie eines Afrikaners namens Bomani, entschließt, diese Möglichkeit nutzbar zu machen. Das Ergebnis dieser Selbsthilfe ist eindrucksvoll (183).

Das letzte Drittel des Buches gilt den ersten Jahren nach Erlangung der Selbständigkeit. Es bietet eine gute Veranschaulichung der gesamtheitlichen Darstellung Bienens, insbesondere $\mathrm{zu}$ den mancherlei Problemen, die die Entscheidung für die Eine Partei aufwirft (zu den Gründen 361; zu den Aufgaben 362) $)^{1}$.

Herbert Krüger

\section{Luis Mercier Vega}

\section{Roads to Power in Latin America}

Transl. by R. Rowland

London Pall Mall Press 1969, X, 208 S. 45 S.

M. V. steht vor dem ewigen Dilemma jeder allgemeinen Untersuchung über Lateinamerika. Er weist selbst nachdrücklich auf die Komplexität und Vielschichtigkeit der sozio-politischen Strukturen Lateinamerikas hin, die keine ge- nerellen Aussagen rechtfertigen, und ist geradezu besessen von der Erkenntnis der Unterschiede und Gegensätze innerhalb des Subkontinentes. Der bloße Einheitsbegriff "Lateinamerika“ sei angesichts der horizontalen und vertikalen Schichtungen, Koexistenz, Úberlagerung, Juxtaposition sozialer Kategorien, von Kulturen, ethnischen Gruppen, ökonomisch-sozialen Klassen usw., die sich ständig und beschleunigt wandeln, fragwürdig geworden. Versagen müßten auch die andernorts entwickelten Kategorien und Begriffsbildungen wie Klassenkampf, Diktatur des Proletariats usw. zur Erfassung dieser Vorgänge. Lateinamerika sei in jeder Hinsicht sui generis. - M. V.'s Analyse jedoch bemüht sich zwar um Differenzierung und vermeidet das Cliché der Klassenkampfanalyse u. ä., hantiert aber doch mit eher traditionellen analytischen Begriffen und muß unvermeidlich ständig verallgemeinern, eingeschränkt durch die halb verlegenen Hinweise auf die besonderen Verhältnisse in diesem oder jenem Lande.

Der zentrale analytische Begriff ist der der Macht, aber mehr als vorausgesetzter Bezugspunkt, ohne daß seine Wirkungsweise gerade in den Verhältnissen Lateinamerikas besonders erläutert würde. Die traditionelle Oligarchie als klassenmäßige Trägerin dieser Macht besteht die Herausforderungen des Wandels in heutiger Zeit - Bevölkerungszuwachs, Migration und Urbanisierung, wirtschaftliche Entwicklung - nicht mehr. Mit oder ohne Revolution - sie ist historisch überholt, hat aber Kultur, Gebärde und Wertvorstellungen der Gesellschaft einschließlich ihrer sozialen Gegner noch unheilvoll geprägt (Geringschätzung manueller Arbeit, Elitebewußtsein usw.). Es gibt aber keine homogene Klasse, die ihre Stelle einnehmen könnte. Die nach traditionellen Begriffen potentiellen Kandidaten Bourgeoisie, sog. Mittelklassen, Bauern, Arbeiter - sind zwar in Teilbereichen vorhanden, aber insgesamt $\mathrm{zu}$ margi-

\footnotetext{
1 Zur augenblicklichen Situation vgl. Krisenzeichen in Tansania, in: Neue Zürcher Zeitung vom 28. April 1971.
} 